\title{
Design and Evaluation of a Collaborative Virtual Environment (CoMove) for Autism Spectrum Disorder Intervention
}

\author{
LIAN ZHANG and QIANG FU, Department of Electrical Engineering \& Computer Science, \\ Vanderbilt University \\ AMY SWANSON, AMY WEITLAUF, and ZACHARY WARREN, Treatment and Research \\ Institute for Autism Spectrum Disorders, Vanderbilt University \\ NILANJAN SARKAR, Department of Mechanical Engineering, Vanderbilt University
}

\begin{abstract}
Autism Spectrum Disorder (ASD) is a neurodevelopmental disorder characterized in part by core deficits in social interaction and communication. A collaborative virtual environment (CVE), which is a computerbased, distributed, virtual space for multiple users to interact with one another and/or with virtual items, has the potential to support flexible, safe, and peer-based social interactions. In this article, we presented the design of a CVE system, called CoMove, with the ultimate goals of measuring and potentially enhancing collaborative interactions and verbal communication of children with ASD when they play collaborative puzzle games with their typically developing (TD) peers in remote locations. CoMove has two distinguishing characteristics: (i) the ability to promote important collaborative behaviors (including information sharing, sequential interactions, and simultaneous interactions) and to provide real-time feedback based on users' game performance; as well as (ii) an objective way to measure and index important aspects of collaboration and verbal-communication skills during system interaction. A feasibility study with 14 pairs -7 ASD/TD pairs and 7 TD/TD pairs-was conducted to initially test the feasibility of CoMove. The results of the study validated the system feasibility and suggested its potential to index important aspects of collaboration and verbal communication.
\end{abstract}

CCS Concepts: • Applied computing $\rightarrow$ Interactive learning environments; $\bullet$ Human-centered computing $\rightarrow$ Collaborative and social computing design and evaluation methods;

Additional Key Words and Phrases: Autism spectrum disorder

ACM Reference format:

Lian Zhang, Qiang Fu, Amy Swanson, Amy Weitlauf, Zachary Warren, and Nilanjan Sarkar. 2018. Design and Evaluation of a Collaborative Virtual Environment (CoMove) for Autism Spectrum Disorder Intervention. ACM Trans. Access. Comput. 11, 2, Article 11 (June 2018), 22 pages.

https://doi.org/10.1145/3209687

This work was supported in part by the National Institutes of Health Grants 1R01MH091102-01A1 and 1R21MH111548-01, National Science Foundation Grant 0967170, and the Hobbs Society Grant from the Vanderbilt Kennedy Center.

Authors' addresses: L. Zhang and Q. Fu, Department of Electrical Engineering \& Computer Science, Vanderbilt University, Nashville, TN 37212; emails: \{lian.zhang, qiang.fu\}@vanderbilt.edu; A. Swanson, A. Weitlauf, and Z. Warren, Treatment and Research Institute for Autism Spectrum Disorders, Vanderbilt University, Nashville, TN 37203; emails: \{amy.r.swanson, amy.s.weitlauf, zachary.e.warren\}@vanderbilt.edu; N. Sarkar, Department of Mechanical Engineering, Vanderbilt University, Nashville, TN 37212; email: nilanjan.sarkar@vanderbilt.edu.

Permission to make digital or hard copies of all or part of this work for personal or classroom use is granted without fee provided that copies are not made or distributed for profit or commercial advantage and that copies bear this notice and the full citation on the first page. Copyrights for components of this work owned by others than ACM must be honored. Abstracting with credit is permitted. To copy otherwise, or republish, to post on servers or to redistribute to lists, requires prior specific permission and/or a fee. Request permissions from permissions@acm.org.

(c) 2018 ACM 1936-7228/2018/06-ART11 $\$ 15.00$

https://doi.org/10.1145/3209687

ACM Transactions on Accessible Computing, Vol. 11, No. 2, Article 11. Publication date: June 2018. 


\section{INTRODUCTION}

\subsection{Background}

Autism Spectrum Disorder (ASD) is a common (1 in 68 U.S. children) and costly (\$3.2 million incremental lifetime care costs) neurodevelopmental disorder characterized in part by core deficits in social interaction and communication [1]. Currently, evidence-based treatments for ASD are difficult for many families to access due to high costs and a limited number of providers. In this capacity, a wide range of studies has investigated the potential of technology, which does not face this same resource shortage, to improve core areas of deficit in ASD [2,3].

The application of interactive technologies, such as virtual environments, may represent a powerful psychometric tool and potential intervention framework. Specifically, providing increased access to immersive, interactive, and safe environments to enhance social communication skills may yield an effective, lower cost treatment paradigm [4,5]. Although research has documented that many children with ASD show an affinity for computer-based interaction [6] and increased engagement in virtual environments [7], most studies of such applications have used preprogrammed confederate agents in a single user mode [8]. This structure of interaction, which is highly dependent on predefined paradigms, is less flexible compared to those in the dynamic real world [9], which may limit their generalizability and therapeutic potential $[6,10]$.

A collaborative virtual environment (CVE) is a computer-based, distributed, virtual space for multiple individuals to interact with one another and/or with virtual items [11]. Compared to existing studies of interaction between children with ASD and computer-controlled virtual avatars $[6,10]$, multiuser CVEs present the opportunity for dynamic user-to-user interactions, instead of user-machine interactions, in shared virtual environments. Such systems, which are suited for collaboration and group work among real users, may offer an effective and beneficial way to foster social relationships among children with ASD and their typically developing (TD) peers $[12,13]$.

Some researchers have evaluated the user-to-user interactions using co-located devices, such as tabletop [14], tablet [15, 16], iPad [17], and wearable devices [18]. These systems allow multiple users to share the same device for face-to-face interactions. However, face-to-face interactions may be initially difficult for children with ASD given the potential multisensory integration deficits associated with ASD [19]. Impairments in multisensory integration, which generally involves how information from the different sensory modalities, such as sight, sound, touch, smell, self-motion, and taste, may be integrated by the nervous system [20], is one of the diagnostic criteria for ASD [21]. In addition, it is difficult to identify users using the co-located devices from a technical point of view [17]. As a result, one user may do all the tasks without collaboration with other users. A distributed system, which allows interactions among users from different locations and reduces information of some sensory modalities, may address these limitations [9, 22]. In what follows, we reviewed previous literature in distributed systems to support user-to-user interactions for ASD intervention.

\subsection{Related Work}

Massively multiplayer online games and social networks have been investigated for ASD intervention [23, 24]. For example, Burke and colleagues analyzed the needs and effects of social communication (such as text-messaging, email, and Facebook) for adults with ASD [25]. They reported many benefits, such as reduced stress and increased greetings. Ringland and colleagues applied the Minecraft game in order to see how individuals with ASD engaged in social play [19]. They found that individuals with ASD were as social as TD individuals in the game. Other multiplayer online games, such as Second-life [8] and Zody's world [17], have also been successfully used to study behaviors of children with ASD in group work. However, researchers usually had limited 
access to the source code of these commercial games. As a result, it could be difficult to alter the games in a way that would allow researchers to structure and investigate specific collaborative activities. In addition, most of these studies analyzed users' behaviors in games using observationbased methods. It will be important to explore other methods that can be used to quantitatively measure interactions between users in these games.

In this context, CVEs with specific social activities have been designed and developed for ASD interventions. Cheng and colleagues designed a virtual restaurant in order to understand empathy in children with ASD [10]. They also developed a CVE with two other social scenes, a classroom scene and an outdoor scene, in order to promote social competence in the ASD population [26]. 3D virtual avatars, which had gestures and facial expressions, were used as representations of real users in the environment, and were applied to investigate the ability of children with ASD to understand these social cues (i.e., gestures and facial expressions) in the environment. iSocial is another important CVE that was designed to investigate social competency in children with ASD [27]. In iSocial, individuals with ASD could interact with each other through the internet using their own 3D virtual avatars. Naturalistic practice learning activities [28] and a social competence curriculum (e.g., facial expression recognition) [29] have been designed as activities in iSocial for understanding social competence. Finally, Wallace and colleagues designed a CVE to teach children with ASD greeting behaviors in a virtual gallery [30]. They found that children with ASD were less sensitive to a negative greeting from the human avatar in the virtual gallery than their TD peers. Although virtual avatars have been successfully used to represent users in these virtual environments [31], the efficacy of the use of avatars for presentation is under debate [11,32]. Please also note that all the activities in these CVEs rely upon specific social skills and are designed to promote external goals of their respective curricula, rather than provide users with game tasks that require and reinforce efficient social interaction as part of the game environment itself.

In addition to these specified social curriculum activities, researchers have also designed collaborative games as interactive activities in CVEs for ASD intervention. Millen and colleagues have developed a CVE with a block party game, which requires users to select the same blocks in order to build a tower, and a talk about the CVE game, which was designed for children with ASD to practice conversation skills [33]. However, these games did not require users to take actions at the same time (i.e., simultaneous interactions). This study reported the preliminary results of a self-report questionnaire, which showed that children with ASD had improved engagement in these CVE-based collaborative games [34]. Schmidt and colleagues have proposed a game-based learning environment for individuals with ASD to learn computational thinking and social skills in groups across the internet [35]. In this environment, users would be able to collaborate with each other and play the Minecraft ${ }^{1}$ video game according to specific collaboration rules. At the time of writing this article, however, results had not been reported.

Although existing massively multiplayer online games and CVEs, which could support flexible interactions between real users from different locations, have been successfully applied for individuals with ASD to practice specific social skills, most of them were not designed to facilitate collaboration, such as taking actions at the same time [11]. Designing systems to facilitate collaboration has two primary challenges. The first challenge is related to the design of collaborative activities themselves in order to foster collaboration. In other words, structuring interactions within a game to require users take collaborative actions, such as taking actions at the same time, sharing information with each other, and playing in order. Collaboration is not something that simply happens whenever users come together [36]. Therefore, carefully designed collaboration strategies are needed in order to enable and encourage collaboration in the environment. The

\footnotetext{
${ }^{1}$ https://education.minecraft.net/.
} 
second challenge is related to evaluating interactions to understand users' collaborative behaviors in specific CVEs and/or validate effects of the CVEs on the users' collaboration and communication skills. Evaluating interactions is challenging, given the unrestricted conversations and complex interactions between real users.

A potential way to address the first challenge in this area is to design collaborative games with embedded collaboration strategies to promote collaboration. A few studies have sought to promote collaboration in the ASD population by designing collaborative games and equipping these games with collaborative strategies using co-located multitouch devices [37, 38]. Gel and colleagues have designed and developed shared storytelling games on co-located multitouch devices to promote collaboration [39]. These games could not only support individual actions but also require simultaneous actions through a collaboration strategy, which require two users to touch and drag items simultaneously in order to move them [40]. Gel and colleagues found that participants initiated more positive social interactions, had more shared play, and performed fewer autistic behaviors while playing these games [41]. Their study also indicated that children with ASD had more positive social interactions and collaborative play in the games with the collaboration strategy than in free-play conditions [15]. Battocchi and colleagues designed collaborative puzzle games with a similar collaboration strategy [14]. They also demonstrated that this kind of puzzle game had positive effects on collaboration in children with ASD. These studies have shown that collaborative games equipped with deliberate collaboration strategies have the potential to promote collaboration in children with ASD. However, these games were developed using co-located multitouch devices instead of distributed CVEs. Co-located multiuser systems can support face-to-face communication with both verbal and non-verbal cues, while communications in distributed CVEs often lack non-verbal cues [42]. Therefore, these existing collaboration strategies cannot be directly used in distributed CVE systems.

In order to address the first challenge, we have developed novel collaborative games with collaborative strategies to promote three important collaborative behaviors, i.e., information sharing, sequential work, and simultaneous work [43-45]. These collaborative behaviors (i.e., sequential work, information sharing, and simultaneous work) were targeted in this study because they relate to a variety of social settings, including employment, education, and game play [46]. Specifically, in group work, people need to be able to take turns (sequential work), decide on certain aspects of the work, and then effectively deliver relevant information (sharing information) and conduct tasks collaboratively (simultaneous work) [12]. Sequential work implies that users take actions one by one (turn taking). Turn taking is a life skill necessary for social success in all environments [47, 48]. Information sharing enables individuals to share resources and knowledge in order to achieve the same goal [44]. Weiss and colleagues observed that information sharing is one of the best ways to train social skills in children with ASD [49]. Information sharing is also important for children with ASD to build friendships with others [47]. Another aspect of group work, i.e., simultaneous work, requires that members of a group work together at the same time [12, 50]. It has been found that the simultaneous interactions could improve social skills by fostering the recognition of the presence of the other, and enhancing interest in partners [41, 51].

The second challenge in this area is to efficiently measure interactions to understand users' within-system behaviors as well as evaluate generalized improvements beyond skill systems and training programs [52]. In order to understand interactions between multiple users, several methods, such as self-report, interviews, observations, performance, and dialog analysis, have been explored by researchers in multiuser systems [53]. Cheng and colleagues used a self-report method and an observation method to evaluate social competence of children with ASD in their CVE [26]. In iSocial [29, 54], the authors analyzed users' behavior by coding their reciprocal social interactions (e.g., conversation initiations, responses, and continuations), identifying their use of available 
avatar-based gestures and movement, and rating their behaviors. However, some aspects of these measurements are task-dependent. Therefore, the methods used to measure social activities in these CVEs cannot be directly applied to evaluate collaborative interactions in our CVE systems. In this article, we have adapted these existing methods to develop a set of metrics for objective measurement of interactions in our CVE-based collaborative games.

\subsection{Current Work}

The primary contributions of this article are as follows: (i) designing a novel computer-based CVE, named CoMove, that can promote important collaborative behaviors, such as sequential work, information sharing, and simultaneous work, between two users in a flexible manner, as well as provide appropriate real-time feedback based on their performance; (ii) providing a potential way to objectively measure both collaborative and communicative behaviors of the users when they play these collaborative games; and (iii) presenting the results of a feasibility study involving seven pairs of ASD/TD and seven pairs of TD/TD children to test system feasibility, and to assess the capacity of CoMove to index important aspects of interactions in the system.

CoMove was designed with the ultimate goals of measuring and potentially enhancing collaborative interactions and verbal communication of children with ASD. In this study, we tested the feasibility of CoMove for children to collaboratively interact and communicate with each other, as well as its potential to index important aspects of the interactions. Testing the system feasibility and its measurement capability lays the groundwork for future investigations into how changes within the system may generalize to real-world interactions. We will evaluate the effects of CoMove on collaborative and verbal-communication skills of children with ASD in their real-world interactions in the future.

The rest of the article is organized as follows. Section 2 presents the development of CoMove with an emphasis on designing collaborative puzzle games to promote collaboration, and generating collaboration- and communication-related data to objectively measure their behaviors. Section 3 provides information about the tasks, participants, and experimental protocol. The results and discussion are presented in Section 4. Finally, Section 5 summarizes the contributions of this article, discusses limitations of the current work, and indicates potential future improvements.

\section{SYSTEM DESIGN}

CoMove is a distributed virtual environment system for two users to communicate and play collaborative puzzle games. The collaborative games in CoMove were designed to promote collaboration through collaborative strategies and to provide feedback based on users' performance. In addition, an objective measurement method was developed for understanding users' collaboration and communication skills within the system. In what follows, we describe (i) the architecture of CoMove, (ii) the characteristics of these collaborative games, and (iii) the details of the objective measurement approach.

\subsection{Architecture of CoMove}

CoMove was designed for two geographically distributed users using their own computers, where each computer is a node of the CVE system, to communicate and collaborate in a shared environment. Given this goal, we designed the architecture shown in Figure. 1, which is divided into a system architecture and an application architecture. The system architecture shows how two nodes (users' computers) are connected and how application data are distributed, while the application architecture is composed of the components used to implement functionalities of the application. 


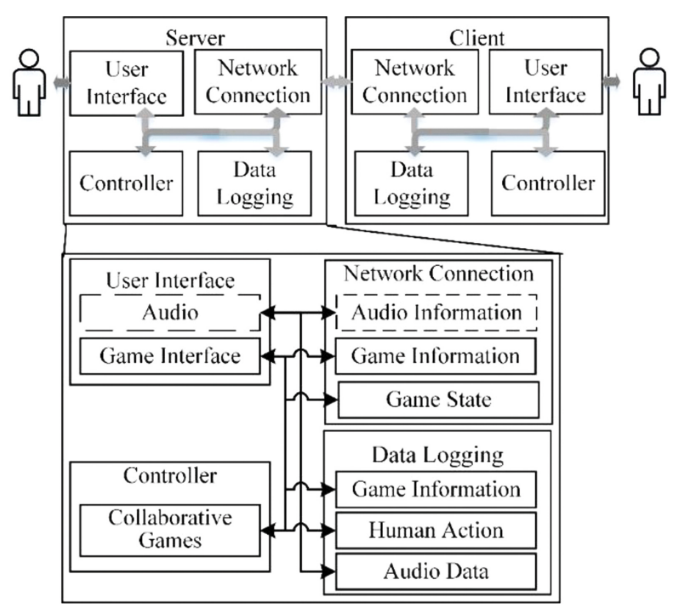

Fig. 1. The architecture of CoMove.

Generally, the design of a CVE system involves (i) how nodes (users' computers) are connected and (ii) how application data are distributed [55]. The design of the system architecture of CoMove aimed to provide an efficient way to address these two issues given specific system requirements. We selected a server-client model for node connection because it is simpler to maintain state consistency compared to the peer-to-peer model [56]. The application data were distributed with a replicated architecture, which replicated the entire application data on each node of CoMove. This replicated architecture can improve the use of network resources since the display data do not need to be transmitted over the network using this architecture [57]. Usually, a scalable CVE system uses a separate central server to store all the application data, distribute specific data to each node, and update games states of all the nodes [58]. Since CoMove targets interactions between two players, no separate central server is necessary at this point. In CoMove, one node is the server, another node is the client, and both nodes have the entire application data. This kind of architecture is convenient and has low network load for a two-player CVE system.

Generally, an application architecture is designed to enable specific functionalities. The applica architecture of CoMove was designed to (i) enable communication and game playing between two geographically distributed users and (ii) understand their behaviors in the system. The application architecture of CoMove has four components: an interface component, a controller component, a network connection component, and a datalogging component. An interface component is generally used to get users' inputs and execute an application's outputs, while a controller component is often used to make decisions and generate responses in computer-based systems [59]. In order to support both verbal communication and game playing, the interface component of CoMove could support both audio input/output (through a microphone and a speaker) and game-related input/output (using a mouse and a graphic display monitor). The controller component of CoMove was implemented using a Finite State Machine (FSM) with the objective to (i) manage multiple collaborative games to facilitate sequential interactions, information sharing, and simultaneous interactions and (ii) provide appropriate performance-based feedback to enhance learning. A network connection component is usually used by a CVE system to connect its multiple nodes [60]. We selected a server-client model to connect distributed nodes for simplicity, as discussed earlier. A datalogging component (called "data collection" in iSocial [54]) is commonly used in CVEs for ASD intervention in order to understand behaviors from the logged data since understanding 


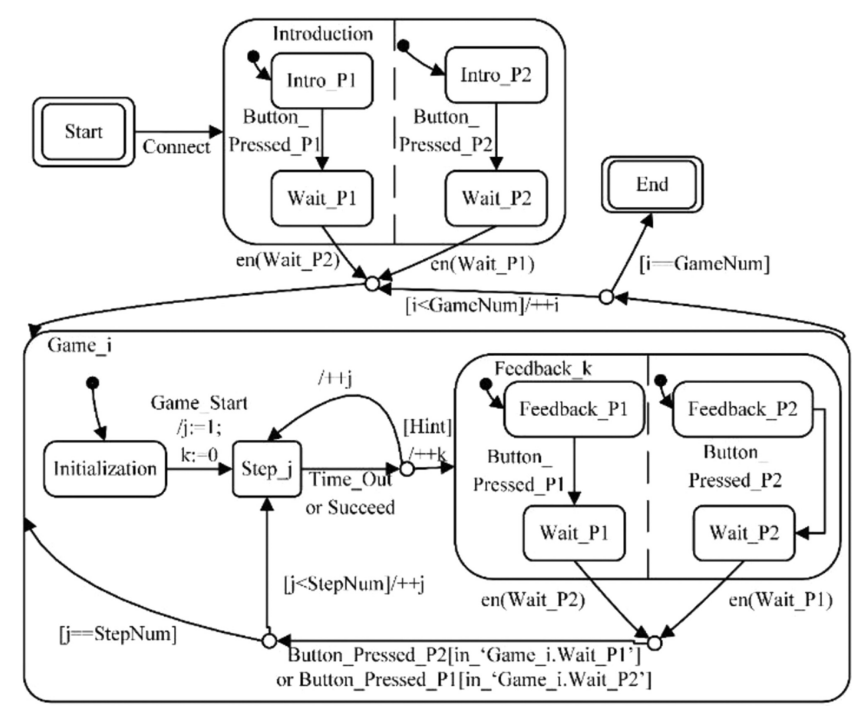

Fig. 2. Finite state machine in the controller component.

behaviors is one of the primary goals in this kind of system. The datalogging component of CoMove could log game information, human actions, and audio data to understand children with ASD's collaborative performance and communication skills in collaborative games. In what follows, we present in detail the development of the controller, network connection, and datalogging components of CoMove.

2.1.1 Controller. The controller component of CoMove was developed in order to manage multiple collaborative puzzle games. We selected collaborative puzzle games as the collaborative activities in CoMove because these games have been widely accepted as engaging children with ASD in collaborative interactions [61], and they are suitable for implementing multiple collaborative strategies to promote collaboration [40]. The logic to manage these collaborative puzzle games is modeled using a FSM with hierarchy and concurrency, as shown in Figure 2.

The Start state of the FSM is the start point of the system. Two players will start their applications in this state and log into the shared environment from their own nodes. Subsequently, an introduction on how to use the environment and how to play the collaborative puzzle games is provided to these players in the Introduction state of the FSM. The Introduction state is a concurrent state, which can not only enable two players to read game information with different reading speeds but also synchronize their states to start a game at the same time. We used a hierarchical state, the Game_ $i$ state, to present a collaborative puzzle game. The Game_ $i$ state has multiple sub-states, such as the Initialization state, the Step_j state, and the Feedback_k state. The system starts a new game when an old one ends. This logic enables players to play multiple collaborative puzzle games that require multiple kinds of collaborative behaviors, i.e., sequential work, information sharing, and simultaneous work. The system can also offer performance-based feedback in the Feedback_k state to help players complete the games.

2.1.2 Network Connection. The network connect component is mainly used to transfer data between different nodes. In CoMove, we designed an efficient data transmission mechanism that used different strategies to transfer different types of data. The trivial game information, such as puzzle piece position, is transferred between the two nodes without synchronization. The game 
state information is transferred using a hand-shaking mechanism for a stable synchronization. The server node of CoMove executes all the computations of the system, such as computing the game states. These computed game states are transferred in seven steps: (1) the client node sends its new data to the server node (this step can be skipped if the new data occurs at the server node);

(2) the server node then computes the new game state; (3) and sends the new game state as a synchronization request to the client node; (4) the client node updates its game state; (5) and sends a synchronization acknowledgment to the server node; (6) after receiving the synchronization acknowledgment from the client node, the server node updates its state; and (7) sends back an acknowledgment to the client node. This hand-shaking mechanism guarantees synchronization between two nodes. The audio data transmission is implemented using the Skype ${ }^{2}$ software for simplicity and stability.

2.1.3 Datalogging. The datalogging component of CoMove is used to store the performanceand communication-related data in order to understand the corresponding within-environment behaviors. The recorded data include game information data as well as player behaviors and audio data during game play. These data are recorded in real time with timestamps, which can be used for offline synchronization.

With these components, CoMove has the capacity to enable collaboration and communication between two geographically distributed players and record data to understand their behaviors in the system. The collaborative puzzle games in CoMove were designed with embedded strategies to promote collaboration. A framework for objective measurement of interactions in CoMove was designed in order to understand important aspects of the within-system behaviors. In what follows, we describe the collaborative puzzle games and the objective measurement framework.

\subsection{Collaborative Puzzle Games}

The puzzle games were designed to promote collaboration in the CVE for users with ASD. Three important collaborative behaviors, i.e., sequential work, information sharing, and simultaneous work, were evaluated. In order to evaluate these three collaborative behaviors, three types of games, turn-taking games, information-sharing games, and collaboration games, were designed.

In each game, players were required to assemble a specific shape by dragging several puzzle pieces following specific rules. In the turn-taking games, each player had full control over the puzzle pieces during his/her turns. In the information-sharing games, colors of some puzzle pieces were hidden for one player while they were visible to the other player. Therefore, the players needed to ask and share the color information in order to move the correct pieces in this type of game. Finally, the collaboration games were implemented with a joint play strategy, which requires two players to drag a puzzle piece in the same direction simultaneously in order to move it. These three types of games together require sequential interactions, simultaneous interactions, and sharing of information. In addition, the system can provide performance-based feedback in these games to help users complete the games. The collaborative puzzle games developed in CoMove were composed of multiple tangram games (Figure 3 (P1_1) and (P2_1) show an instance of tangram games) and a castle-building game (shown in Figure 3 (P1_3) and (P2_3)).

In each tangram game, players were required to assemble a specific shape from seven flat pieces. Each game had seven steps. In each step, a single puzzle piece needed to be moved to its target position. A total of seven tangram games were designed with five configuration features: (1) who can see the colors of all the puzzle piece; (2) who can move puzzle pieces; (3) who can rotate puzzle pieces; (4) the maximum time duration of a step; and (5) the feedback information. The values of

\footnotetext{
${ }^{2}$ skype.com.
} 


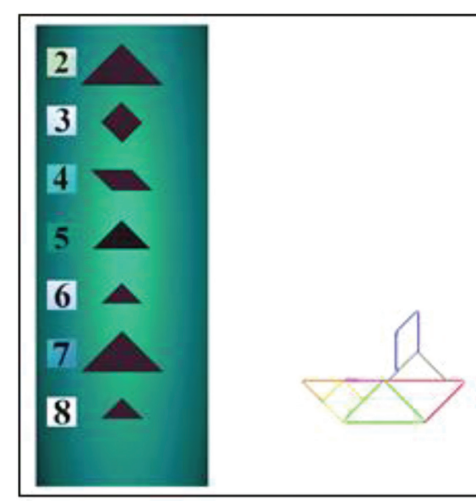

(P1_1)

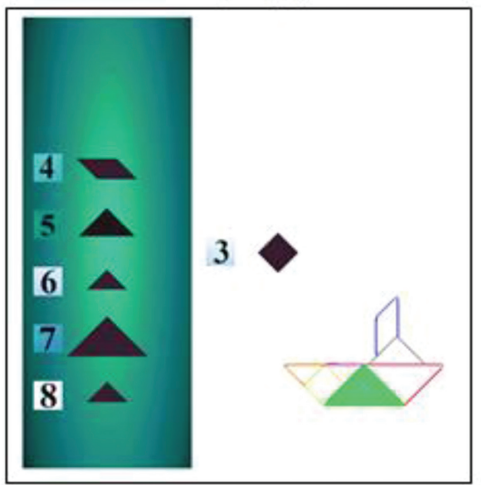

(P1_2)

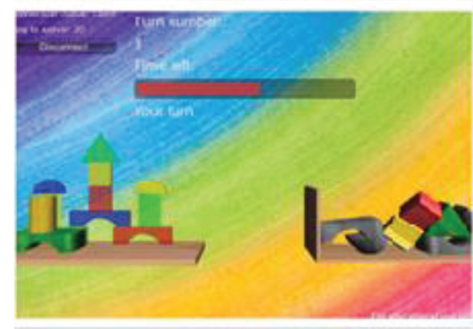

(P1_3)

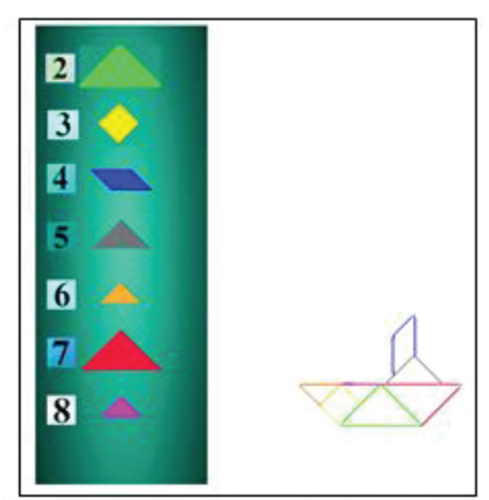

(P2_1)
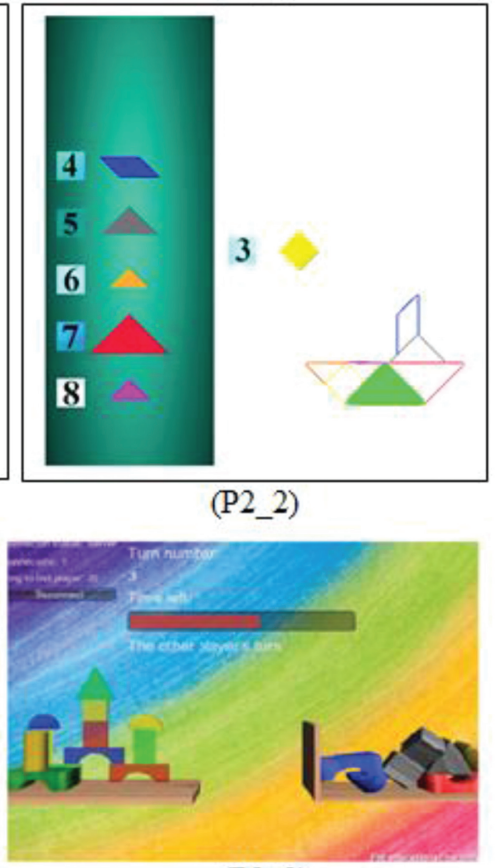

(P2_3)

Fig. 3. P1_1 and P2_1 are screenshots of a tangram game captured from two CVE nodes; P1_2 and P2_2 are screenshots of the same tangram game from the nodes after two pieces being moved; and P1_3 and P2_3 are screenshots of a castle game of the nodes.

the first four features of each game are listed in Table 1. For example, in game T1, both P1 and P2 can see the color of puzzle pieces. In this game, P1 and P2 could move puzzle pieces one by one, and the puzzle pieces could rotate automatically. The maximum time duration for a player to successfully move a puzzle piece in game T1 is 30 seconds. If the player failed to move any puzzle piece within the time duration, the system automatically moved a puzzle piece to its target position. The fifth feature, i.e., feedback information, is about how to move or rotate pieces in the game. For example, an example of feedback information in the collaboration game, T3, is "Maybe 


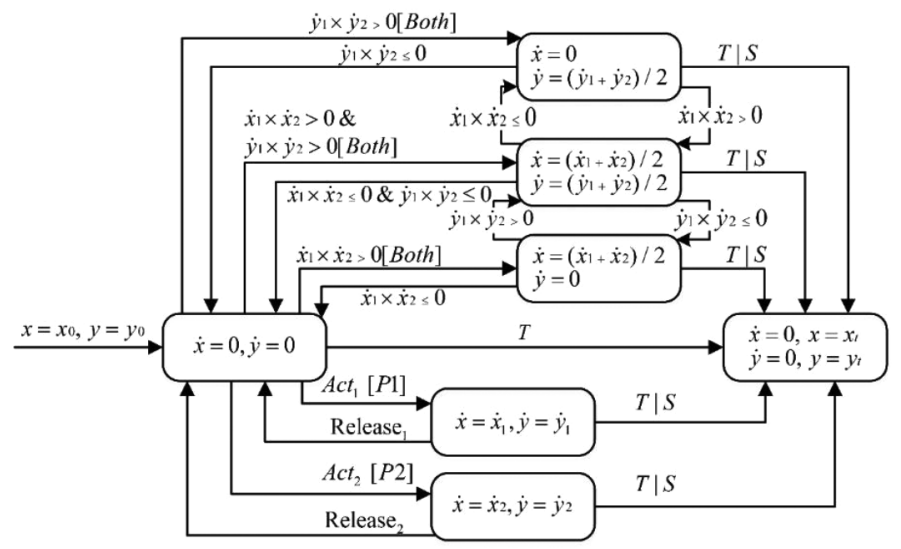

Fig. 4. The hybrid automaton.

Table 1. The Value of the Configuration Features for Each Game

\begin{tabular}{|l|l|l|c|c|}
\hline Game name & $\begin{array}{c}\text { Who can see the } \\
\text { colors of the puzzle } \\
\text { pieces }\end{array}$ & $\begin{array}{c}\text { Who can move the } \\
\text { puzzle pieces }\end{array}$ & $\begin{array}{c}\text { Who can rotate } \\
\text { the puzzle pieces }\end{array}$ & $\begin{array}{c}\text { Time } \\
\text { duration } \\
\text { (seconds) }\end{array}$ \\
\hline T1 & P1 $^{*}$ and P2* & $\begin{array}{l}\text { P1 in step 1, 3, 5, 7; } \\
\text { P2 in step 2, 4,6 }\end{array}$ & Auto* & 30 \\
\hline T2 & P1 and P2 & $\begin{array}{l}\text { P1 in step 3, 4, 7; } \\
\text { P2 in step 1, 2, 5, 6 }\end{array}$ & Auto & 30 \\
\hline T3 & P1 and P2 & $\begin{array}{l}\text { P1 and P2 in all } \\
\text { steps }\end{array}$ & Auto & 45 \\
\hline T4 & P1 & P2 in all steps & P2 & 40 \\
\hline T5 & P2 & P1 in all steps & P1 & 40 \\
\hline T6 & P1 & $\begin{array}{l}\text { P1 and P2 in all } \\
\text { steps }\end{array}$ & P2 & 50 \\
\hline T7 & P2 & $\begin{array}{l}\text { P1 and P2 in all } \\
\text { steps }\end{array}$ & P1 & 50 \\
\hline
\end{tabular}

${ }^{*} P 1$ means the first player, and P2 means the second player.

${ }^{* *}$ Auto means the puzzle pieces will be rotated automatically by the system.

two people are needed to move this puzzle piece." In a tangram game, feedback information is offered when players fail the first two steps and the first four steps of the game.

The main focus of these games is to facilitate information sharing as well as promote both sequential and simultaneous interactions. The information sharing was facilitated by hiding information for one of the two users. For example, in game T4, the first player can see colors of all puzzle pieces, while the second player can move all the pieces. Therefore, the first player needs to share color information with the second player in order for the second player to move the correct puzzle pieces.

In order to promote both sequential and simultaneous interaction, we developed a collaboration strategy, which requires two players to move puzzle pieces individually or simultaneously in order to complete a specific game. Previous studies using multitouch devices for co-located multiuser interactions implemented the collaboration strategy by defining rules, such as holding on 
Table 2. Symbols Used in the Hybrid Automaton and Their Descriptions

\begin{tabular}{|c|c|c|}
\hline Symbol & Type & Description \\
\hline $\operatorname{Act}_{i}, i=1,2$ & Event & $\begin{array}{l}\text { The first }(i=1) \text { or the second }(i=2) \text { player takes actions on } \\
\text { the puzzle piece }\end{array}$ \\
\hline $\begin{array}{l}\text { Release }_{i}, i=1, \\
2\end{array}$ & Event & $\begin{array}{l}\text { The first }(i=1) \text { or the second }(i=2) \text { player release the puzzle } \\
\text { piece }\end{array}$ \\
\hline$\overline{P_{i}, i=1,2}$ & Condition & $\begin{array}{l}\text { The first }(i=1) \text { or the second }(i=2) \text { player's turn to move } \\
\text { puzzle pieces (turn-taking games) }\end{array}$ \\
\hline Both & Condition & $\begin{array}{l}\text { Two players move puzzle pieces together (collaboration } \\
\text { games) }\end{array}$ \\
\hline$\overline{\mathrm{T}}$ & Event & Time out \\
\hline $\bar{S}$ & Event & Succeed \\
\hline$x$ & Variable & Shift of a puzzle piece in the horizontal direction \\
\hline $\bar{y}$ & Variable & Shift of a puzzle piece in the vertical direction \\
\hline$x_{o}$ & Variable & Initial position of a puzzle piece in the horizontal direction \\
\hline$\underline{y_{o}}$ & Variable & Initial position of a puzzle piece in the vertical direction \\
\hline$x_{t}$ & Variable & Target position of a puzzle piece in the horizontal direction \\
\hline$\overline{y_{t}}$ & Variable & Target position of a puzzle piece in the vertical direction \\
\hline$\dot{\dot{x}}$ & Variable & Moving speed of a puzzle piece in the horizontal direction \\
\hline$\dot{\bar{y}}$ & Variable & Moving speed of a puzzle piece in the vertical direction \\
\hline$\dot{\bar{x}}_{i}, i=1,2$ & Variable & $\begin{array}{l}\text { Dragging speed of a puzzle piece by the ith player in the } \\
\text { horizontal direction }\end{array}$ \\
\hline$\dot{y}_{i}, i=1,2$ & Variable & $\begin{array}{l}\text { Dragging speed of a puzzle piece by the ith player in the } \\
\text { vertical direction }\end{array}$ \\
\hline
\end{tabular}

the same puzzle piece [41, 62]. Those co-located systems enable players to communicate with each other in a face-to-face mode, which makes it easy to follow these rules. It is challenging to implement natural face-to-face communication in distributed CVE systems from a technical point of view [42]. Interactions in distributed systems lack natural non-verbal cues (such as gestures and eye contacts). This fact makes it harder for users to follow these rules in distributed CVE systems. Therefore, we developed a different way to implement this collaboration strategy in our distributed CVE system in order to fulfill the requirements to promote both sequential and simultaneous interactions.

The hybrid automaton is used to represent how a puzzle piece responds when two players take actions in different games. All the symbols and their descriptions of the hybrid automaton are show in Table 2. In a collaboration game, the condition Both is true in the hybrid automaton. Under this condition, for example, if two players drag the puzzle piece toward the same direction $\left(\dot{x}_{1} \times \dot{x}_{2}>0\right)$, the velocity of the puzzle piece in the horizontal direction is $\left(\dot{x}_{1}+\dot{x}_{2}\right) / 2$. If one player drags the piece to the right and the other player drags it to the left $\left(\dot{x}_{1} \times \dot{x}_{2}<0\right)$, or one player stops dragging in the horizontal direction $\left(\dot{x}_{1} \times \dot{x}_{2}=0\right)$, the velocity of the puzzle piece in the horizontal direction is 0 . When the puzzle piece is successfully moved to its target area (presented with the event $\mathrm{S}$ ) or the time reaches the maximum time duration (presented with the event $\mathrm{T}$ ), the position of the puzzle piece is automatically set to $\left(x_{t}, y_{t}\right)$. Using this hybrid automaton model, players are forced to interact differently, i.e., dragging individually or dragging simultaneously, in different games. 
Table 3. The Game Information of the Castle Game

\begin{tabular}{|l|l|l|l|l|l|l|l|l|l|l|}
\hline Index of a puzzle piece & 1 & 2 & 3 & 4 & 5 & 6 & 7 & 8 & 9 & 10 \\
\hline Who can move the puzzle pieces & P1 & P1 & P1 & P2 & P2 & P1 & P1 & P2 & P2 & P2 \\
\hline Who can see the color of the puzzle pieces & P2 & P2 & P2 & P1 & P1 & P2 & P2 & P1 & P1 & P1 \\
\hline
\end{tabular}

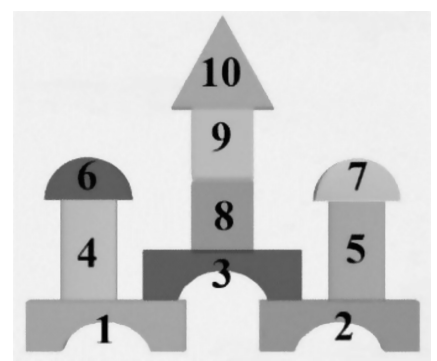

Fig. 5. The index of each piece in the castle game.

The castle-building game, as shown in Figure 3 P1_3 and P2_3, is another CoMove game, which can enable turn-taking and information sharing as well provide performance-based feedback. The game is also designed based on the five configuration features mentioned in the tangram game. Each player could only see the colors of five pieces and could only move the other five pieces, which are summarized in Table 3 using the (i) who can move the puzzle pieces and (ii) who can see the colors of the puzzle pieces. P1 in Table 3 means the first player and P2 means the second player. The index of each puzzle piece is shown in Figure 5. These puzzle pieces can be automatically rotated when they are correctly located. The maximum time duration without successfully moving a puzzle piece is 30 seconds. The castle game was different from the tangram games since its pieces had gravity and therefore needed to be built in order. In summary, players can move the pieces in turns with order constraints in the castle game. A player may have no piece to move during his/her turn because of the order constraints. Thus, a feedback is displayed for the player to skip his/her turn when no piece is movable for the player.

\subsection{Objective Measurement Method}

Existing literature lacks standardized methods for objective measurements in CVE-based collaborative games. Measurements of collaboration and communication in CVE systems may be taskdependent. Therefore, measurement methods used for existing CVE systems with social activities cannot be directly used in our CVE system with the collaborative puzzle games. One of the important goals of this article is to understand the collaboration and verbal-communication skills of children with ASD when they play collaborative games with their TD peers in CoMove. In order to understand the collaborative interactions and verbal communication in the system, three kinds of data (game information data, human behaviors data, and audio data) were recorded. The real-time recorded game information data included the start time, the end time, and the success frequency of a game. The human behaviors data included information about dragging a piece, rotating a piece, and releasing a piece. The verbal communication of each user in the environment was recorded as the audio data. These recorded data were analyzed offline based on several selected performance measures and communication measures.

The performance measures, as shown in Table 4, were chosen such that they could directly reflect the effects of the collaborative puzzle games and the collaboration strategies on users' collaborative behaviors within the system. For example, the measure-collaborative movement ratio was 
Table 4. All the Performance- and Communication-Related Measures

\begin{tabular}{|c|c|c|}
\hline & Measure name & Measure description \\
\hline 1 & Success frequency & How many times an individual succeeded in moving pieces in game(s) \\
\hline 2 & $\begin{array}{l}\text { Collaborative movement } \\
\text { ratio }\end{array}$ & $\begin{array}{l}\text { The ratio of the time duration of a piece being moved by two individuals } \\
\text { simultaneously to the time duration of an individual dragged the piece }\end{array}$ \\
\hline 3 & Frequency of words & How many words per minute an individual spoke \\
\hline 4 & $\begin{array}{l}\text { Frequency of asking } \\
\text { question }\end{array}$ & $\begin{array}{l}\text { How often (the number of the utterance per minute) an individual asked } \\
\text { task-related questions }\end{array}$ \\
\hline 5 & $\begin{array}{l}\text { Frequency of information } \\
\text { sharing-response }\end{array}$ & How often an individual responded to task-related information \\
\hline 6 & $\begin{array}{l}\text { Frequency of information } \\
\text { sharing-spontaneous }\end{array}$ & How often an individual initiated a task-related information \\
\hline 7 & $\begin{array}{l}\text { Frequency of social } \\
\text { reinforcement-positive }\end{array}$ & How often an individual used positive social reinforcement, such as "good job" \\
\hline 8 & $\begin{array}{l}\text { Frequency of social } \\
\text { reinforcement-negative }\end{array}$ & How often an individual used negative social feedback, such as "stupid" \\
\hline 9 & Frequency of directives & How often an individual directed other individuals to tack action \\
\hline 10 & $\begin{array}{l}\text { Frequency of } \\
\text { social-oriented utterance }\end{array}$ & $\begin{array}{l}\text { How often an individual used social-oriented utterance, such as "what is your } \\
\text { name?" }\end{array}$ \\
\hline
\end{tabular}

defined as the ratio of collaborative movement time (i.e., how long two users move puzzle pieces together) to total time (i.e., how long an individual moves puzzle pieces). The values of these performance measures were automatically computed offline from the recorded game information and human behavior data.

The communication measures, also shown in Table 4, were selected based on both previous studies in peer-based interactions [63] and the specific tasks in our environment. Previous studies have utilized several communication measures, including the number of spoken words [63], asking questions and answering questions [64], information sharing [65], positive reinforcement and negative reinforcement [66], and directive utterances [67], to understand both ASD and TD children in peer-based interactions. Based on these studies, we defined a corresponding seven communication measures to understand users' conversations about the game play in CoMove. Although the majority of conversations in multiuser interactions are task-oriented, non-task-oriented conversations (or social-oriented conversations) are also used by the users during interactions [68]. The nontask-oriented conversations were recorded using a frequency of social-oriented utterance measure. In order to index important aspects of communication in CoMove, we analyzed recorded audio data based on these measures by the following steps: (i) transcribe the recorded audio data using the DragonNaturallySpeaking software (www.nuance.com), (ii) correct the transcription by two native speakers of English, and (iii) classify the corrected transcription into these communication measures by a human coder, who was blind with respect to the tasks and the participants.

In order to mitigate coding variability, our team of clinical psychologists and engineers collaborated to develop a rule-based coding protocol with structured instructions on how to classify these communication measures in a consistent manner. We provide two examples of predefined rules here. One, if the utterance starts with "what," "which," "do," "is," and "are," it is classified as a question-asking utterance. Two, if an utterance provides color, position, rotation, direction, and puzzle piece information, it is an information-sharing utterance. In addition, if the information-sharing utterance has the same information as its preceding utterance, it is an information-sharing-response utterance. Otherwise, it is an information-sharing-spontaneous utterance. Please note that the human coder was trained and directly supervised by two licensed 
Table 5. Characteristics of the Participants

\begin{tabular}{ccccc}
\hline & Age Mean (SD) & Gender Female/male & SRS-2 total raw score Mean (SD) & SCQ current total score Mean (SD) \\
\hline ASD & $13.71(2.70)$ & $1 / 6$ & $107(22.35)$ & $19(9.40)$ \\
TD1 & $13.89(3.14)$ & $1 / 6$ & $13.71(16.06)$ & $1.29(1.38)$ \\
TD2 & $10.59(2.00)$ & $2 / 12$ & $18.14(16.60)$ & $2.14(3.53)$ \\
\hline
\end{tabular}

clinical psychologists who specialize in ASD intervention. The coder had been trained for behavior coding across a series of other related works [69, 70]. By recording and analyzing these data from both performance and communication measures, this article provides a way to objectively measure collaboration and communication children with ASD within CoMove.

\section{FEASIBILITY STUDY}

\subsection{Participants}

A total of 28 children, 7 age- and gender-matched ASD/TD pairs and 7 age- and gender-matched TD/TD pairs (age range: 7-17 years) were recruited to participate. The rationale for the above grouping is as follows: (i) the ultimate goal of the study is to understand and enhance the collaborative interaction and communication skills of children with ASD with their TD peers; and (ii) we also wanted to explore how two TD children interact under the same conditions so that we can identify meaningful differences between TD and ASD interactions. All the children with ASD had a clinical diagnosis of ASD from a licensed clinical psychologist, an IQ higher than 70, and the ability to use phrased speech as determined by a trained therapist.

To assess current levels of ASD symptoms across groups, the Social Responsiveness Scale, second edition (SRS-2) [71] and Social Communication Questionnaire (SCQ) [72] were completed by the parents of participants in both groups. These scales are efficient quantitative measures of interpersonal behavior, communication, and repetitive/stereotypic behavior characteristic of ASD. SRS-2 is an objective measure of symptoms associated with ASD. A total T-score of 76 or higher is considered strongly associated with clinical diagnosis of ASD. T-scores of 66-75 are interpreted as moderate deficiencies in reciprocal social behavior, whereas a T-score of 60-65 is interpreted as a mild range. Total scores in the range of 59T and below are generally not associated with clinically significant ASD. SCQ is a parent-reported screening measure that taps the symptomatology associated with ASD [72]. The SCQ has two versions: Lifetime and Current. A Lifetime Total Score above 15 suggests that the individual is likely to have ASD. The Current Total Score is used to measure the individual's behaviors during the most recent 3-month period.

The characteristics of the 28 participants are shown in Table 5. TD1 represents the TD children in the ASD/TD pairs, while TD2 represents the TD children in the TD/TD pairs. These participants did not know each other before their experiments. They could not see each other but could talk with each other during the experiments through audio chat. The experiments were approved by the Vanderbilt University Institutional Review Board (IRB).

\subsection{Tasks and Protocol}

Each pair of participants took part in a 50-minute-long session where the participants sat in two different rooms in the same building. Two Dell desktop computers T3610 (E5-220 V3 CPU and 8GB RAM) were used as two nodes of CoMove. The connection of two nodes in these rooms was created via a Local Area Network (LAN). The experimental session included a pre-test, followed by a game playing session, and then a post-test. At the very beginning of an experiment, participants 
Table 6. The Games and Their Order During One Experiment

\begin{tabular}{l|l}
\hline Pre-test & Castle game, T6, T7 \\
\hline Game playing & T1, T2, T3, T4, T5, T6, T7, T7, T6, T5, T4 \\
\hline Post-test & Castle game, T6, T7 \\
\hline
\end{tabular}

were instructed that they would be playing three different kinds of games in CoMove. However, they did not receive detailed instructions on the objective of each game. The participants were encouraged to communicate with each other in order to find out how to play each game. Feedback was also provided when necessary, as discussed in Section 2.2, in order to help participants play these games. After the introduction, the participants completed a pre-test that consisted of three baseline games (one castle game and two tangram games). The castle game is a turn-taking game requiring information sharing, while the two tangram games are enforced-collaboration games, T6 and T7, which are defined in Table 1. The target shapes of T6 and T7 in the pre-test were different from those in the game playing session. Eleven tangram games were included in the game playing session, which lasted approximately 30 minutes. After the game playing session, the three baseline games were presented again to serve as the post-test. The games and the order of games during the experiment are shown in Table 6. During the experiment, we recorded both video and audio of the participants and their computer screens. The participants were informed that both audio and video recordings of the experiment would be made.

\section{RESULTS AND DISCUSSIONS}

\subsection{System Performance}

Overall, CoMove worked as designed. All participants completed their experiments with a zerodropout rate. The system mostly ran at 60 frames per second and had an average network delay of 1 millisecond or less when used through a LAN. The system could successfully log the performance and audio data of the participants as well as game information data via the datalogging component. The audio data were recorded for each game and each individual. A total of 467 audio files were recorded from 28 participants (17 audio files for each participant). One audio file was recorded incorrectly for unknown reasons. For the incorrectly recorded audio file, we manually extracted the corresponding audio data from the recorded video of that experiment. These results support the system feasibility. Specifically, the system is able to support communication and collaboration between children with ASD and their TD peers, as well as to record related data for meaningful measurements.

In order to index important aspects of interactions in CoMove, we analyzed changes of the predefined performance and communication measures (these measures are discussed in Section 2.3) from the pre-test to the post-test for each group. Specifically, we statistically compared the results of pre- and post-tests regarding these predefined performance and communication measures for all participants. The Wilcoxon Signed-rank test [73] was used for the statistical analysis with 0.05 as the alpha level. Given the limited power corresponding to this small sample size and conservative non-parametric approach, we also examined effect sizes using Spearman's rank correlation ( 0.1 is small effect, 0.3 is medium effect, and 0.5 is large effect) [74]. Although analyzing the differences between children with ASD and their TD peers in CVEs may be important, this article focuses on understanding changes of collaboration and verbal-communication skills within the system. Therefore, we compared the changes of each predefined measure from the pre-test to the post-test for each group. 
Table 7. Performance Results from Pre-Tests to Post-Tests

\begin{tabular}{cc|ccc|cccc}
\hline & & \multicolumn{3}{|c|}{ Castle game } & \multicolumn{3}{c}{ Tangram games } \\
\hline & Measure & Pre-test & Post-test & $\rho$ & Pre-test & Post-test & $\rho$ \\
\hline ASD & 1 & 2 & 5 & 0.81 & 7 & 14 & $0.18^{*}$ \\
$N=7$ & 2 & - & - & - & 0.11 & 0.22 & $0.30^{*}$ \\
\hline TD1 & 1 & 3 & 4 & $0.87^{*}$ & 7 & 14 & $0.18^{*}$ \\
$N=7$ & 2 & - & - & - & 0.11 & 0.12 & $0.86^{*}$ \\
\hline TD2 & 1 & 1 & 3.5 & $0.76^{* *}$ & 8 & 12 & 0.30 \\
\cline { 2 - 8 }$N=14$ & 2 & - & - & - & 0.13 & 0.09 & 0.22 \\
\hline
\end{tabular}

*indicates $p<0.05$ and **indicates $p<0.001$.

\subsection{Results}

The statistical analysis results for each subject group from the pre-test to the post-test across all performance measures are shown in Table 7 with the $\rho$ columns showing all effect sizes. Overall, participants in all groups (i.e., children with ASD, TD1 children, and TD2 children) demonstrated statistically significant improvements on some performance measures from pre- to posttest. Wilcoxon Signed-rank test indicated a significantly higher collaborative movement ratio in the post-test relative to the pre-test in tangram games for children with ASD $(p<0.05, \rho=0.30)$ and TD1 $(p<0.05, \rho=0.86)$. ASD and TD1 also had a significantly higher success frequency in tangram games (two participants of a pair shared the same value regarding this frequency) in the post-test compared to the pre-test ( $p<0.05, \rho=0.18$ ). In addition, Wilcoxon Signed-rank test indicated a significantly increased success frequency in the castle game regarding TD1 $(p<0.05$, $\rho=0.87)$ and TD2 $(p<0.001, \rho=0.76)$.

Table 8 summarizes the statistical differences from the pre-test to the post-test across all communication measures for all participants, while the $\rho$ columns show all effect size results. Overall, all participants had changes in some communication measures from the pre-test to the post-test, although not all of these changes were statistically significant. The word frequency of children with ASD in the castle game in the post-test is higher that the frequency in the pre-test, but not at a statistically significant level $(p=0.25, \rho=0.60)$. TD1 had a significantly higher word frequency $(p<0.05, \rho=0.78)$ in the castle game in the post-test compared to the pre-test. For children with ASD, the frequencies of asking questions and information sharing-spontaneous are higher in the post-test compared to the pre-test; however, these differences did not achieve statistical significance. TD1 children had a significantly higher frequency of information sharing-response in the castle game $(p<0.05, \rho=0.29)$.

In summary, children with ASD, TD1 children, and TD2 children demonstrated improvements regarding some important collaboration and verbal-communication measures from the pre-test to the post-test, although not all improvements were statistically significant. In the next Section 4.3, we further discuss the results of children with ASD.

\subsection{Discussion}

In spite of its small sample size and lack of an active comparison condition, results from this pilot study indicated that children with ASD showed improvements on some collaborative performance measures when using our system. In CoMove, children with ASD had a significantly increased success frequency in tangram games in the post-test compared to the pre-test. These results are consistent with previous work by Bauminger-Zviely et al. [75], who had reported that collaborative games could improve collaborative performance of children with ASD. Battocchi et al. [76] 
Table 8. The Communication Measures Results

\begin{tabular}{|c|c|c|c|c|c|c|c|}
\hline & & \multicolumn{3}{|c|}{ Castle game } & \multicolumn{3}{|c|}{ Tangram games } \\
\hline & Measure & Pre-test & Post-test & $\rho$ & Pre-test & Post-test & $\rho$ \\
\hline \multirow{8}{*}{ ASD $N=7$} & 3 & 0.79 & 0.910 & 0.60 & 0.90 & 0.88 & 0.68 \\
\hline & 4 & 0.02 & 0.014 & 0.84 & $8 e-3$ & 0.02 & 0.67 \\
\hline & 5 & 0.05 & 0.069 & 0.46 & 0.05 & 0.04 & 0.89 \\
\hline & 6 & 0.02 & 0.025 & 0.78 & 0.02 & 0.04 & 0.54 \\
\hline & 7 & 0 & 0 & 0.45 & 0 & 0 & 0.15 \\
\hline & 8 & 0 & 0 & - & 0 & 0 & 1 \\
\hline & 9 & 0.01 & 0.014 & 0.52 & 0.01 & 0.02 & 0.59 \\
\hline & 10 & 0 & 0 & -0.17 & 0 & 0 & - \\
\hline \multirow{8}{*}{ TD1 $N=7$} & 3 & 0.73 & 1.038 & $0.78^{*}$ & 0.90 & 0.92 & 0.86 \\
\hline & 4 & 0.03 & 0.035 & 0.68 & 0.01 & 0.01 & 0.86 \\
\hline & 5 & 0.05 & 0.097 & $0.29^{*}$ & 0.05 & 0.03 & 0.29 \\
\hline & 6 & 0.01 & 0.023 & 0.78 & 0.02 & 0.03 & 0.68 \\
\hline & 7 & 0 & 0 & - & $2 e-3$ & 0 & 0.45 \\
\hline & 8 & 0 & 0 & - & 0 & 0 & - \\
\hline & 9 & $6 e-3$ & 0 & 0.61 & $8 \mathrm{e}-3$ & 0.03 & 0.68 \\
\hline & 10 & 0 & 0 & - & 0 & 0 & - \\
\hline \multirow{8}{*}{ TD2 $N=14$} & 3 & 0.74 & 0.863 & 0.81 & 0.75 & 0.58 & $0.58^{*}$ \\
\hline & 4 & 0.01 & 0.020 & 0.40 & 0.02 & 0.01 & 0.72 \\
\hline & 5 & 0.05 & 0.069 & 0.81 & 0.05 & 0.04 & 0.69 \\
\hline & 6 & 0.01 & 0.018 & 0.65 & 0.01 & 0.03 & 0.67 \\
\hline & 7 & 0 & 0 & -0.16 & $6 e-3$ & 0 & $0.46^{\star}$ \\
\hline & 8 & 0 & 0 & - & 0 & 0 & 0.18 \\
\hline & 9 & $6 e-3$ & $8 e-3$ & 0.14 & 0.02 & 0.02 & 0.63 \\
\hline & 10 & 0 & 0 & $0.83^{*}$ & 0 & 0 & $0.07^{\star}$ \\
\hline
\end{tabular}

*indicates $P<0.05$.

mentioned that enforced-collaboration games, which required simultaneous activities, could be used in collaboration training of children with ASD. We observed a statistically significant increase in the collaborative movement ratio for children with ASD. These results support the potential usability of the collaboration games to promote collaboration in the ASD population in the future.

In both castle and tangram games, children with ASD asked more questions in the post-test compared to the pre-test, even though the difference was not statistically significant. We noticed that in the pre-test of tangram games, the question-asking frequency of children with ASD, $M d n=8 e-3$, was lower than the frequency of TD1 children, $M d n=0.01$, and TD2 children, $M d n=0.02$. This is consistent with the findings of Schmidt et al. [9], which showed that children with ASD had a lower frequency of initiations, including question asking. However, in the post-test of our CVE puzzle games, the question-asking frequency of children with ASD, $M d n=0.012$, was comparable to the frequency of TD1 children, $M d n=0.012$, and TD2 children, $M d n=0.011$. This result may be in line with Owen-Schryver's findings, which suggested that children with ASD could make more initiations after interacting with their TD peers in peer-mediated interactions [77]. However, these differences did not achieve statistical significance. The effects of CoMove on verbalcommunication skills of children with ASD need to be further investigated in the future.

We also found that in both castle and tangram games, the success frequency of children with ASD was comparable to the frequency of TD children. The potential reason is that all participants 
with ASD had average IQ and phrase speech. Please note that this is not true for all children with ASD, some of whom have intellectual disability or severe language impairment. Therefore, these results should not be considered representative of how all children with ASD would perform.

Based on the above results and discussion, we cautiously conjecture that CoMove has the potential to index important aspects of interactions in the system. The effects of CoMove on collaborative and verbal-communication skills of children with ASD need to be further investigated in the future using a long-term, multisession skill transfer study with more subjects. We believe that the results of this preliminary study warrant further exploration on the potential impacts of CoMove on participants' skills in real life in a clinical study of generalization.

\section{CONCLUSIONS, LIMITATION, AND FUTURE WORK}

This article presents the design and development of a novel CVE system, CoMove, and the results of a preliminary feasibility study with 28 participants (7 ASD/TD pairs and 7 TD/TD pairs) using CoMove. A CVE system allows peer-based interaction in a shared and controlled virtual environment. The CVE system in this article has two distinguishing characteristics. First, it applies collaborative puzzle games and collaboration strategies to promote important collaborative behaviors, i.e., information sharing, sequential interactions, and simultaneous interactions. Second, it provides a potential way to objectively measure important aspects of collaboration and communication of these children when they play collaborative puzzle games in the CVE.

A total of $7 \mathrm{ASD} / \mathrm{TD}$ pairs and $7 \mathrm{TD} / \mathrm{TD}$ pairs were involved in a preliminary feasibility study to initially test system functionality, and determine its capacity for indexing important aspects of within-system interactions. Regarding functionality, all participants completed their experiments with a zero-dropout rate. Additionally, all system software and hardware worked as designed, capturing necessary data with minimal loss. We also observed and measured changes in participant collaborative behaviors. Specifically, we observed statistically significant changes on some important performance measures in children with ASD: a significantly increased success frequency and a significantly increased collaborative movement ratio in tangram games. We also observed changes (although not statistically significant) in communication measures in the children with ASD, such as an increased frequency of question asking in tangram games. These results support that our system has the potential to index important aspects of interactions in the system.

While the present work is promising, several limitations exist in the current work. We designed CoMove with the ultimate goals of measuring and potentially enhancing collaborative interactions and verbal communication of children with ASD. In this article, we tested the system feasibility and its potential to measure important aspects of within-system interactions using a preliminary study. In the next step, we will evaluate how the within-system interactions correlate with and potentially impact participants' skills in real life in a clinical study of generalization. This future work will include long-term, multisession experiments and many more participants. These participants will play real-world collaborative tasks as baseline tasks before and after interacting within CoMove. Their behavior changes in the baseline tasks will be measured using multiple measurement methods, such as questionnaires, observation, and evaluation tools [53], and used to indicate the impact of CoMove.

Second, only one coder coded the participants' communication behaviors in this study. In the current protocol, we were forced to rely on a single coder due to resource limitations in the laboratory of our behavioral collaborators in this preliminary work. In order for the coder to code communication behaviors in a consistent manner, our team of clinical psychologists and engineers collaborated to create predefined rules, as discussed in Section 2.3. However, the reliability of the coding results still needs to be evaluated using multiple coders and establishing high inter-rater reliability. We plan to use multiple coders in future work replicating and extending these findings. 
Third, finding ways to provide and encourage face-to-face communication is a challenge for all CVEs. CoMove, at present, can only support audio chat, which simplified the data analysis with an emphasis on the verbal communication in this preliminary study. Other non-verbal communication modes such as eye contact and gestures will be introduced in the future via a video chat, an eye tracker, and a gesture recognition method.

Fourth, CoMove was tested in a LAN. In order to broaden its applicability, the system will need to be tested in a global area network in the future. Asymmetric latencies are issues associated with the server-client architecture. While the asymmetric latencies between the server node and the client node were small in the LAN, the asymmetric latencies need to be addressed in order for CoMove to be used in a global area network.

Finally, the current system included performance-based feedback. In the future, other kinds of feedback, such as communication-based feedback, will be included to foster more social-oriented communication. Despite the above-mentioned limitations, we believe that the present work, which offers a potential way to address current challenges of CVEs for ASD intervention and provides important preliminary insights in CVE and collaborative games-based intervention, makes a compelling case in this research area.

\section{REFERENCES}

[1] C. G. Snoek, M. Worring, and A. W. Smeulders. 2005. Early versus late fusion in semantic video analysis. In Proceedings of the 13th Annual ACM International Conference on Multimedia, 399-402.

[2] M. L. Sundberg and J. W. Partington. 1998. Teaching language to children with autism and other developmental disabilities. Behavior Analysts Inc., Pleasant Hill, CA.

[3] N. Bauminger. 2002. The facilitation of social-emotional understanding and social interaction in high-functioning children with autism: Intervention outcomes. Fournal of Autism and Developmental Disorders 32 (2002), 283-298.

[4] D. Strickland. 1997. Virtual reality for the treatment of autism. Studies in Health Technology and Informatics (1997), 81-86.

[5] C. Mei, L. Mason, and J. Quarles. 2015. How 3D virtual humans built by adolescents with ASD affect their 3D interactions. In Proceedings of the 17th International ACM SIGACCESS Conference on Computers \& Accessibility, 155-162.

[6] D. Moore, Y. Cheng, P. McGrath, and N. J. Powell. 2005. Collaborative virtual environment technology for people with autism. Focus on Autism and Other Developmental Disabilities 20(2005), 231-243.

[7] U. Lahiri, E. Bekele, E. Dohrmann, Z. Warren, and N. Sarkar. 2015. A physiologically informed virtual reality based social communication system for individuals with autism. fournal of Autism and Developmental Disorders 45 (2015), 919-931.

[8] N. Newbutt. 2014. Representations of self in classroom virtual worlds: A case-study of pupils on the autism spectrum. Game-Based Learning: Challenges and Opportunities, Cambridge Scholars Publishing (2014), 165.

[9] M. Schmidt, J. Laffey, and J. Stichter. 2011. Virtual social competence instruction for individuals with autism spectrum disorders: Beyond the single-user experience. In Proceedings of CSCL, 816-820.

[10] Y. Cheng, H.-C. Chiang, J. Ye, and L.-h. Cheng. 2010. Enhancing empathy instruction using a collaborative virtual learning environment for children with autistic spectrum conditions. Computers \& Education 55 (2010), 1449-1458.

[11] S. Benford, C. Greenhalgh, T. Rodden, and J. Pycock. 2001. Collaborative virtual environments. Communications of the ACM 44 (2001), 79-85.

[12] P. J. Leman. 2015. How do groups work? Age differences in performance and the social outcomes of peer collaboration. Cognitive Science 39 (2015), 804-820.

[13] S. Reynolds, R. M. Bendixen, T. Lawrence, and S. J. Lane, 2011. A pilot study examining activity participation, sensory responsiveness, and competence in children with high functioning autism spectrum disorder. Fournal of Autism and Developmental Disorders 41 (2011), 1496-1506.

[14] A. Battocchi, F. Pianesi, D. Tomasini, M. Zancanaro, G. Esposito, P. Venuti, A. Ben Sasson, E. Gal, and P. L. Weiss. 2009. Collaborative puzzle game: A tabletop interactive game for fostering collaboration in children with autism spectrum disorders (ASD). In Proceedings of the ACM International Conference on Interactive Tabletops and Surfaces, 197-204.

[15] A. Ben-Sasson, L. Lamash, and E. Gal. 2013. To enforce or not to enforce? The use of collaborative interfaces to promote social skills in children with high functioning autism spectrum disorder. Autism 17 (2013), 608-622.

[16] J. P. Hourcade, N. E. Bullock-Rest, and T. E. Hansen. 2012. Multitouch tablet applications and activities to enhance the social skills of children with autism spectrum disorders. Personal and Ubiquitous Computing 16 (2012), 157168. 
[17] L. E. Boyd, K. E. Ringland, O. L. Haimson, H. Fernandez, M. Bistarkey, and G. R. Hayes. 2015. Evaluating a collaborative iPad game's impact on social relationships for children with autism spectrum disorder. ACM Transactions on Accessible Computing (TACCESS) 7 (2015), 3.

[18] L. E. Boyd, A. Rangel, H. Tomimbang, A. Conejo-Toledo, K. Patel, M. Tentori, and G. R. Hayes. 2016. SayWAT: Augmenting face-to-face conversations for adults with autism. In Proceedings of the 2016 CHI Conference on Human Factors in Computing Systems, 4872-4883.

[19] K. E. Ringland, C. T. Wolf, H. Faucett, L. Dombrowski, and G. R. Hayes. 2016. Will I always be not social?: Reconceptualizing sociality in the context of a minecraft community for autism. In Proceedings of the 2016 CHI Conference on Human Factors in Computing Systems, 1256-1269.

[20] B. E. Stein, T. R. Stanford, and B. A. Rowland. 2009. The neural basis of multisensory integration in the midbrain: Its organization and maturation. Hearing Research 258 (2009), 4-15.

[21] J. C. McPartland, B. Reichow, and F. R. Volkmar. 2012. Sensitivity and specificity of proposed DSM-5 diagnostic criteria for autism spectrum disorder. Fournal of the American Academy of Child \& Adolescent Psychiatry 51 (2012), 368-383.

[22] L. Millen, S. Cobb, H. Patel, and T. Glover. 2012. Collaborative virtual environment for conducting design sessions with students with autism spectrum conditions. In Proceedings of the 9th International Conference on Disability, Virtual Reality and Associated Technologies, 269-278.

[23] S. Caltagirone, M. Keys, B. Schlief, and M. J. Willshire. 2002. Architecture for a massively multiplayer online role playing game engine. Journal of Computing Sciences in Colleges 18 (2002), 105-116.

[24] D. Livingstone, J. Kemp, and E. Edgar. 2008. From multi-user virtual environment to 3D virtual learning environment. ALT-7: Research in Learning Technology 16 (2008), 139-150.

[25] M. Burke, R. Kraut, and D. Williams, 2010. Social use of computer-mediated communication by adults on the autism spectrum. In Proceedings of the 2010 ACM Conference on Computer Supported Cooperative Work, 425-434.

[26] Y. Cheng and J. Ye. 2010. Exploring the social competence of students with autism spectrum conditions in a collaborative virtual learning environment-The pilot study. Computers \& Education 54 (2010), 1068-1077.

[27] J. Laffey, M. Schmidt, K. Galyen, and J. Stichter. 2012. Smart 3D collaborative virtual learning environments: A preliminary framework. Journal of Ambient Intelligence and Smart Environments 4 (2012), 49-66.

[28] X. Wang, J. Laffey, W. Xing, Y. Ma, and J. Stichter. 2016. Exploring embodied social presence of youth with autism in 3D collaborative virtual learning environment: A case study. Computers in Human Behavior 55 (2016), 310-321.

[29] J. P. Stichter, J. Laffey, K. Galyen, and M. Herzog, 2014. iSocial: Delivering the social competence intervention for adolescents (SCI-A) in a 3D virtual learning environment for youth with high functioning autism. Fournal of Autism and Developmental Disorders 44 (2014), 417-430.

[30] S. Wallace, S. Parsons, and A. Bailey. 2017. Self-reported sense of presence and responses to social stimuli by adolescents with ASD in a collaborative virtual reality environment. Fournal of Intellectual and Developmental Disability 42 , 2 (2017), 131-141.

[31] E. S. Liu and G. K. Theodoropoulos. 2014. Interest management for distributed virtual environments: A survey. ACM Computing Surveys (CSUR) 46, 4 (2014), Article 51.

[32] S. Natkin and C. Yan. User model in multiplayer mixed reality entertainment applications. In Proceedings of the 2006 ACM SIGCHI International Conference on Advances in Computer Entertainment Technology, 85.

[33] L. Millen, T. Hawkins, S. Cobb, M. Zancanaro, T. Glover, P. L. Weiss, and E. Gal. Collaborative technologies for children with autism. In Proceedings of the 10th International Conference on Interaction Design and Children, 246-249.

[34] P. L. Weiss, E. Gal, M. Zancanaro, L. Giusti, S. Cobb, L. Millen, T. Hawkins, T. Glover, D. Sanassy, and S. Eden. 2011. Usability of technology supported social competence training for children on the autism spectrum. In Proceedings of the 2011 International Conference on Virtual Rehabilitation (ICVR '11), 1-8.

[35] M. Schmidt and D. Beck. 2016. Computational thinking and social skills in virtuoso: An immersive, digital gamebased learning environment for youth with autism spectrum disorder. In Proceedings of the International Conference on Immersive Learning, 113-121.

[36] P. Dillenbourg. 2002. Over-scripting CSCL: The risks of blending collaborative learning with instructional design. Heerlen, Open Universiteit Nederland.

[37] M. Nakano, S. Sato, K. Komatani, K. Matsuyama, K. Funakoshi, and H. G. Okuno. 2011. A two-stage domain selection framework for extensible multi-domain spoken dialogue systems. In Proceedings of the SIGDIAL 2011 Conference, $18-29$.

[38] H. A. M. Noor, F. Shahbodin, and N. C. Pee. 2012. Serious game for autism children: Review of literature. World Academy of Science, Engineering and Technology, International fournal of Social, Behavioral, Educational, Economic, Business and Industrial Engineering 6 (2012), 554-559.

[39] N. Bauminger, D. Goren-Bar, E. Gal, P. L. Weiss, J. Kupersmitt, F. Pianesi, O. Stock, and M. Zancanaro. 2007. Enhancing social communication in high-functioning children with autism through a co-located interface. In Proceedings IEEE 9th Workshop on of Multimedia Signal Processing (MMSP '07), 18-21. 
[40] A. Cappelletti, G. Gelmini, F. Pianesi, F. Rossi, and M. Zancanaro. Enforcing cooperative storytelling: First studies. In Proceedings of the IEEE International Conference on Advanced Learning Technologies, 281-285.

[41] E. Gal, N. Bauminger, D. Goren-Bar, F. Pianesi, O. Stock, M. Zancanaro, and P. L. T. Weiss. 2009. Enhancing social communication of children with high-functioning autism through a co-located interface. AI \& Society 24 (2009), 7584.

[42] M. M. Montoya, A. P. Massey, and N. S. Lockwood. 3D collaborative virtual environments: Exploring the link between collaborative behaviors and team performance. Decision Sciences 42 (2011), 451-476.

[43] N. Rummel and H. Spada. 2005. Learning to collaborate: An instructional approach to promoting collaborative problem solving in computer-mediated settings. The Journal of the Learning Sciences 14 (2005), 201-24.

[44] D. W. Johnson and R. T. Johnson. 2008. Cooperation and the use of technology. Handbook of Research on Educational Communications and Technology: A Project of the Association for Educational Communications and Technology. Routledge.

[45] E. Gal, D. Goren-Bar, E. Gazit, N. Bauminger, A. Cappelletti, F. Pianesi, O. Stock, M. Zancanaro, and P. Weiss. 2005. Enhancing social communication through story-telling among high-functioning children with autism. Intelligent Technologies for Interactive Entertainment. Springer, 320-323.

[46] S. W. White, K. Keonig, and L. Scahill. 2007. Social skills development in children with autism spectrum disorders: A review of the intervention research. Journal of Autism and Developmental Disorders 37 (2007), 1858-1868.

[47] P. A. Rao, D. C. Beidel, and M. J. Murray. 2008. Social skills interventions for children with asperger's syndrome or high-functioning autism: A review and recommendations. fournal of Autism and Developmental Disorders 38 (2008), $353-361$.

[48] V. Bernard-Opitz, N. Sriram, and S. Nakhoda-Sapuan. 2001. Enhancing social problem solving in children with autism and normal children through computer-assisted instruction. Journal of Autism and Developmental Disorders 31 (2001), 377-384.

[49] M. J. Weiss and S. L. Harris. 2001. Teaching social skills to people with autism. Behavior Modification 25 (2001), $785-$ 802.

[50] E. Gal, D. Goren-Bar, E. Gazit, N. Bauminger, A. Cappelletti, F. Pianesi, O. Stock, M. Zancanaro, and P. Weiss, 2005. Enhancing social communication through story-telling among high-functioning children with autism. In Proceedings of the International Conference on Intelligent Technologies for Interactive Entertainment, 320-323.

[51] M. Zancanaro, F. Pianesi, O. Stock, P. Venuti, A. Cappelletti, G. Iandolo, M. Prete, and F. Rossi. 2007. Children in the museum: An environment for collaborative storytelling. PEACH-Intelligent Interfaces for Museum Visits. Springer, $165-184$.

[52] E. Anagnostou, N. Jones, M. Huerta, A. K. Halladay, P. Wang, L. Scahill, J. P. Horrigan, C. Kasari, C. Lord, and D. Choi. 2015. Measuring social communication behaviors as a treatment endpoint in individuals with autism spectrum disorder. Autism 19 (2015), 622-636.

[53] C. L. Gress, M. Fior, A. F. Hadwin, and P. H. Winne. 2010. Measurement and assessment in computer-supported collaborative learning. Computers in Human Behavior 26 (2010), 806-814.

[54] M. Schmidt, J. M. Laffey, C. T. Schmidt, X. Wang, and J. Stichter. 2012. Developing methods for understanding social behavior in a 3D virtual learning environment. Computers in Human Behavior 28 (2012), 405-413.

[55] C. Fleury, T. Duval, V. Gouranton, and B. Arnaldi. 2010. Architectures and mechanisms to maintain efficiently consistency in collaborative virtual environments. In Proceedings of SEARIS 2010 (IEEE VR 2010 Workshop on Software Engineering and Architectures for Realtime Interactive Systems).

[56] M. R. Macedonia and M. J. Zyda. 1997. A taxonomy for networked virtual environments. IEEE Multimedia 4, 1 (1997) $48-56$.

[57] D. D. Suthers. 2001. Architectures for computer supported collaborative learning. In Proceedings of the IEEE International Conference on Advanced Learning Technologies. 25-28.

[58] L. Gautier and C. Diot. 1998. Design and evaluation of MiMaze a multi-player game on the internet. In Proceedings of the IEEE International Conference on Multimedia Computing and Systems. 233-236.

[59] A. Dix. 2009. Human-Computer Interaction. Springer.

[60] J. Bowers, J. Pycock, and J. O’Brien. 1996. Talk and embodiment in collaborative virtual environments. In Proceedings of the SIGCHI Conference on Human Factors in Computing Systems. 58-65.

[61] A. Battocchi, A. Ben-Sasson, G. Esposito, E. Gal, F. Pianesi, D. Tomasini, P. Venuti, P. Weiss, and M. Zancanaro. 2010. Collaborative puzzle game: A tabletop interface for fostering collaborative skills in children with autism spectrum disorders. Journal of Assistive Technologies 4 (2010), 4-13.

[62] M. Fan, A. N. Antle, C. Neustaedter, and A. F. Wise. 2014. Exploring how a co-dependent tangible tool design supports collaboration in a tabletop activity. In Proceedings of the 18th International Conference on Supporting Group Work. 8190 .

[63] S. D. Teasley. 1995. The role of talk in children's peer collaborations. Developmental Psychology 31 (1995), 207. 
[64] C. Van Boxtel, J. Van der Linden, and G. Kanselaar. 2000. Collaborative learning tasks and the elaboration of conceptual knowledge. Learning and Instruction 10 (2000), 311-330.

[65] D. D. Curtis and M. J. Lawson. 2001. Exploring collaborative online learning. fournal of Asynchronous Learning Networks 5 (2001), 21-34.

[66] C. M. Mitchell, E. Y. Ha, K. E. Boyer, and J. C. Lester. 2013. Learner characteristics and dialogue: Recognising effective and student-adaptive tutorial strategies. International fournal of Learning Technology 25, 8 (2013), 382-403.

[67] S. Caballé, T. Daradoumis, F. Xhafa, and A. Juan, 2011. Providing effective feedback, monitoring and evaluation to on-line collaborative learning discussions. Computers in Human Behavior 27 (2011), 1372-1381.

[68] M. H. Charlop-Christy, M. Carpenter, L. Le, L. A. LeBlanc, and K. Kellet. 2002. Using the picture exchange communication system (PECS) with children with autism: Assessment of PECS acquisition, speech, social-communicative behavior, and problem behavior. Journal of Applied Behavior Analysis 35 (2002), 213-231.

[69] C. Liu, P. Agrawal, N. Sarkar, and S. Chen. 2009. Dynamic difficulty adjustment in computer games through real-time anxiety-based affective feedback. International fournal of Human-Computer Interaction 25 (2009), 506-529.

[70] L. Zhang, J. Wade, D. Bian, J. Fan, A. Swanson, A. Weitlauf, Z. Warren, and N. Sarkar. 2017. Cognitive load measurement in a virtual reality-based driving system for autism intervention. IEEE Transactions on Affective Computing 8 (2017), 176-189.

[71] J. N. Constantino and C. P. Gruber. 2002. The Social Responsiveness Scale. Western Psychological Services, Los Angeles.

[72] M. Rutter, A. Bailey, and C. Lord, The Social Communication Questionnaire: Manual: Western Psychological Services, Los Angeles.

[73] J. D. Gibbons and S. Chakraborti. 2011. Nonparametric Statistical Inference. Springer.

[74] J. Cohen. 1988. Statistical Power Analysis for the Behavioral Sciences. Lawrence Earlbaum Associates, Hillsdale, NJ, 20-26.

[75] N. Bauminger-Zviely, S. Eden, M. Zancanaro, P. L. Weiss, and E. Gal. 2013. Increasing social engagement in children with high-functioning autism spectrum disorder using collaborative technologies in the school environment. Autism 17 (2013), 317-339.

[76] G. F. Wilson and C. A. Russell. 2007. Performance enhancement in an uninhabited air vehicle task using psychophysiologically determined adaptive aiding. Human Factors: The fournal of the Human Factors and Ergonomics Society 49 (2007), 1005-1018.

[77] J. S. Owen-DeSchryver, E. G. Carr, S. I. Cale, and A. Blakeley-Smith. 2008. Promoting social interactions between students with autism spectrum disorders and their peers in inclusive school settings. Focus on Autism and Other Developmental Disabilities 23 (2008), 15-28.

Received April 2017; revised January 2018; accepted April 2018 